\title{
Generadores y transversalidad del discurso sobre inmigración en el ámbito metropolitano de Sevilla ${ }^{1}$
}

\author{
Ricardo Iglesias-Pascual. Universidad Pablo de Olavide, Sevilla, España.
}

RESUMEN | Los problemas generalizados de integración del inmigrante ponen de manifiesto la importancia de desarrollar un análisis holístico de las dinámicas subyacentes en la movilidad residencial, segregación e integración de dicha población. En este sentido, disciplinas como el análisis del discurso pueden representar una importante aportación, tanto para el estudio de las causas y consecuencias de la segregación residencial, como para un adecuado diseño de políticas de integración del inmigrante. Por ello, mediante un enfoque cualitativo se analiza la generación, expansión y efectos del discurso social sobre inmigración en el territorio metropolitano de Sevilla. Los resultados muestran cómo, a pesar del importante papel de los medios de comunicación en la difusión de este discurso, se deben tener en cuenta otros elementos, como la visión de los residentes y exresidentes, o los desplazamientos ocasionales a los barrios de inmigrantes, a la hora de entender cómo se construye el imaginario sobre la inmigración y su papel en la integración y segregación residencial.

PALABRAS CLAVE | segregación, migración, imaginarios urbanos.

ABSTRACT | Problems related to immigrant integration reveal the importance of developing a holistic analysis of the underlying dynamics of residential mobility, segregation and integration. In this sense, aspects such as discourse analysis can represent an important contribution to both the analysis of causes and consequences of residential segregation, as to a proper design of inclusive immigration public policy. Through a qualitative analysis, this study focuses on the development of a social discourse, along with its expansion and effects on immigration in Seville's Metropolitan Area. The results show that, despite the important role of the media in the diffusion of this speech, other factors such as the vision of residents and former residents, or occasional travel, should be taken into account to understand how immigration imaginary and the role it plays in the integration and residential segregation, is built.

KEYWORDs | segregation, migration, urban imaginary.

Recibido el 8 de julio de 2015, aprobado el 22 de abril de 2016

E-mail: riglpas@upo.es

1 El núcleo central de este artículo es fruto de la participación en el I Simposium Internacional EDIso Estudios sobre Discurso y Sociedad, celebrado en julio de 2014 en Sevilla, y se enmarca dentro del Proyecto de Excelencia del Plan Nacional de I+D+I "Dinámicas y transformaciones territoriales, funcionales y sociales de las áreas metropolitanas españolas en un horizonte de sostenibilidad” (cso2014-55780-C3-1-P), financiado por el Ministerio de Economía y Competitividad, Espańa. 


\section{Introducción}

La inmigración por motivos económicos se ha convertido, desde finales del siglo xx, en una variable sociodemográfica clave en la Unión Europea, y sociedades posfordistas en general, a la hora de entender sus principales dinámicas sociales. En este sentido, es en la ciudad en mayor grado donde la inmigración se visualiza y se hace presente, especialmente en aquellos barrios donde la población extranjera alcanza mayores niveles de concentración. Este proceso suele ir asociado a la aparición de actitudes xenófobas, debido a la generación de sentimientos intersubjetivos de invasión y competencia social por los recursos (Farley, Steeh, Krysan, Jackso \& Reeves, 1994; Herranz de Rafael et al., 2012, Iglesias Pascual, 2015a, 2015b).

En esta línea, en la presente investigación se considera que los miedos y estereotipos subyacentes en la xenofobia se encuentran en la base del discurso generado sobre la inmigración en el espacio metropolitano. Este discurso, originado y personalizado inicialmente en los barrios con altas proporciones de población inmigrante, finalmente, y de manera intersubjetiva, se hace extensible a toda la ciudad. De este modo se plasma en un imaginario social colectivamente recreado, el cual tiene su reflejo espacial en la activación y desarrollo del proceso de segregación residencial que experimenta la población inmigrante extranjera, especialmente aquellas nacionalidades con escasos recursos económicos, clasificadas como de inmigrantes económicos (Iglesias Pascual, 2015; 2016).

Ante el importante papel que desempeñan el discurso social y la subjetividad espacial en las dinámicas residenciales de la población inmigrante y en su integración al ámbito urbano, este artículo se centra en los principales elementos generadores del discurso socialmente construido sobre aquellos barrios del ámbito metropolitano sevillano con altas densidades de población inmigrante, y en la transversalidad territorial de dicho discurso. Es decir, el análisis aborda cómo se genera, distribuye y modifica el discurso sobre la inmigración en las diferentes escalas del ámbito metropolitano. En este sentido, se considera que las variaciones discursivas sobre la inmigración constituyen una importante fuente de datos para identificar los principios implícitos en el origen y desarrollo de los procesos de segregación residencial; y que, a la vez, pueden ofrecer una importante información para el diseño de las políticas de integración de las comunidades inmigrantes.

Para ejemplificar esta propuesta analítica, se ha centrado el estudio en el discurso sobre la inmigración generado en Sevilla, como ejemplo de área metropolitana consolidada española. Sevilla, cuarta ciudad española por número de habitantes según los datos del Padrón municipal de 2011, con una población de 703.021 habitantes, presentaba un porcentaje de población extranjera de 5,4\%, cifra baja en relación con el resto de las grandes ciudades españolas. ${ }^{2}$ Sin embargo, pese a este escaso número de extranjeros, los índices de segregación de las distintas nacionalidades la sitúan como una de las ciudades con valores de segregación más elevados

2 En 2011, Barcelona era la ciudad con mayor presencia de extranjeros (17,23\%), seguida de Madrid (16,99\%) y Valencia (13,76\%). Incluso se puede observar que ciudades de menor tamańo poblacional que Sevilla, como son los casos de Zaragoza (12,86\%) o Málaga (8,44\%), presentan mayores porcentajes de población extranjera. 
del país (Palacios \& Vidal, 2014; Vono \& Bayona, 2011). Este hecho hace especialmente interesante el análisis del discurso sobre la inmigración en la ciudad a la hora de explicar la divergencia entre los valores de población extranjera y los índices de segregación. Para ello, a través de lo que hemos denominado 'territorialización del discurso' (Iglesias Pascual, 2016), metodología basada en la realización de grupos de discusión (GD) y el análisis social del discurso, se ha indagado en los elementos que se encuentran en la base del discurso socialmente construido sobre los barrios con alta presencia de inmigración y su evolución en las diferentes escalas del territorio metropolitano.

A la hora de desarrollar la propuesta analítica, en primer lugar se exponen brevemente los principios teóricos en los que se basa la investigación. Tras una recapitulación sobre el contexto epistemológico y socioespacial de partida, se desarrolla una breve descripción de la metodología aplicada. Posteriormente se explicitan y analizan los principales elementos generadores del discurso social sobre la inmigración, para pasar posteriormente al análisis de la transversalidad de las diferentes categorías discursivas en el espacio metropolitano de Sevilla. Por último, a modo de conclusión, se reflexiona sobre las implicaciones que el análisis generado puede presentar en el diseño de políticas de integración del inmigrante en la ciudad.

\section{Contextualización epistemológica y territorial de la investigación}

Este artículo se enmarca en una investigación más amplia centrada en el análisis de los efectos de la subjetividad socioespacial en el proceso de segregación residencial de la población inmigrante extranjera. El punto de partida de dicha propuesta es el desarrollo teórico de lo que hemos denominado 'concepciones espaciales subjetivas' (de aquí en adelante, CES) y 'espacio inducido'. A partir de estos elementos conceptuales se analiza cómo la recreación del imaginario socioespacial determina y relaciona directamente procesos como la segregación residencial, xenofobia y filtrado residencial en aquellos barrios que albergan mayor presencia de población extranjera (Iglesias Pascual, 2015, 2016). Dentro de este esquema analítico, las CES se proponen como un constructo intersubjetivo que engloba todas aquellas variables que desarrollan la subjetividad espacial, poniendo siempre el foco de atención en el punto de vista del sujeto. Se plantea cómo el sujeto, de manera individual aunque determinado por el contexto social, en su proceso de reconstrucción del espacio no discrimina entre diferentes categorías analíticas, como hace el investigador, sino que construye la subjetividad espacial como un todo, donde saca información de lo percibido, lo imaginado, lo simbólico, etcétera. Con dicha información se da forma a un discurso social que, individual e intersubjetivamente, explicita la manera de percibir los diferentes territorios de la ciudad; en este caso, una ciudad cada vez más extensa territorialmente y difícilmente abarcable, donde adquiere una creciente importancia lo que se ha denominado como espacio inducido (Iglesias Pascual, 2016). Dicha dimensión del espacio sería el fruto de las informaciones extraídas diariamente de los medios de comunicación, las interrelaciones sociales y los imaginarios sociales. El análisis de esta dimensión inducida del espacio, ante el progresivo aumento de los ámbitos ignotos en un espacio metropolitano caracterizado por su 
carácter difuso y extenso en el territorio, resulta de creciente interés a la hora de entender los diferentes comportamientos socioespaciales.

El elemento clave para determinar cómo se construyen dichas CES y su efecto sobre el proceso de segregación residencial de la población extranjera, es el discurso socioespacial. Dicho discurso y su análisis resultan fundamentales para entender las implicaciones espaciales que tienen las diferentes actitudes generadas hacia la inmigración (Iglesias Pascual, 2014). En este sentido, la relación entre el discurso social, las opiniones y actitudes que se construyen hacia la población inmigrante y el tratamiento de los medios de comunicación, ha sido analizada extensamente (Cea D’Ancona, 2007 y 2009; Van Dyjk, 2003; Igartua, Otero, Muñiz \& Gómez, 2006; Igartua, Muñiz, Otero \& De la Fuente, 2007).

En el presente artículo se plantea profundizar en la dimensión metropolitana de la construcción del discurso sobre el inmigrante, matizando los enfoques anteriores y añadiendo otros elementos a la hora de examinar su formación respecto de aquellos barrios que albergan mayores porcentajes de población inmigrante. Para ello se analizan tanto los principales generadores discursivos detectados, como el funcionamiento de su difusión por el espacio metropolitano. El estudio del discurso sobre la inmigración, su distribución y transversalidad, además de ayudar a entender los mecanismos subyacentes en el proceso de filtrado residencial de la población autóctona y la consecuente segregación residencial, puede otorgar herramientas para un mejor diseño de las políticas de integración del inmigrante en el barrio y en el conjunto de la ciudad.

Para dar cuerpo a todo lo hasta aquí planteado epistemológicamente, se hace necesario concretar el contexto socioterritorial de la investigación. Tal como se dijo en el apartado introductorio, al darse en una comunidad porcentajes de población extranjera superiores al $20 \%$ se produce la aparición de actitudes xenófobas entre la población (Farley et al., 1994; 1994; Herranz de Rafael et al., 2012). Sobre la base de este criterio se seleccionaron en Sevilla aquellos barrios que en 2011 albergaban secciones censales con porcentajes de extranjeros iguales o superiores al $20 \%{ }^{3}$ Fundamento de esta decisión metodológica es la consideración de la construcción de actitudes xenófobas como proceso social intersubjetivo que desempeńa un papel fundamental en el origen y extensión del proceso de segregación residencial (Iglesias Pascual, 2015) y estigmatización territorial (Wacquant, 2007, pp. 195 y ss), especialmente en barrios con altas densidades de población extranjera procedente de países de bajo nivel de renta. En el caso de Sevilla, se diferenciaron tres zonas cuyas secciones censales alcanzaban porcentajes iguales o superiores al 20\% (figuras 1 y 2).

3 A la hora de seleccionar los ámbitos de estudio se desestimó utilizar otros índices de medición de la segregación intraurbana, como el Coeficiente de Localización (Brown \& Chung, 2006) o la distribución de Tipos Residenciales de Jhonston, Forrest y Poulsen (2002), ya que estos analizan la proporción de la población extranjera respecto a la sociedad de acogida mostrando las zonas de mayor concentración de extranjeros, pero no identifican aquellos ámbitos que la sociedad de acogida identifica como "barrios de inmigrantes" y que están en el origen del discurso sobre la inmigración en la ciudad. Para más detalles sobre este aspecto aplicado a las principales ciudades españolas, véase Iglesias (2015b). 
FIGURA I | Sevilla. Porcentaje de población extranjera por secciones censales, 2011

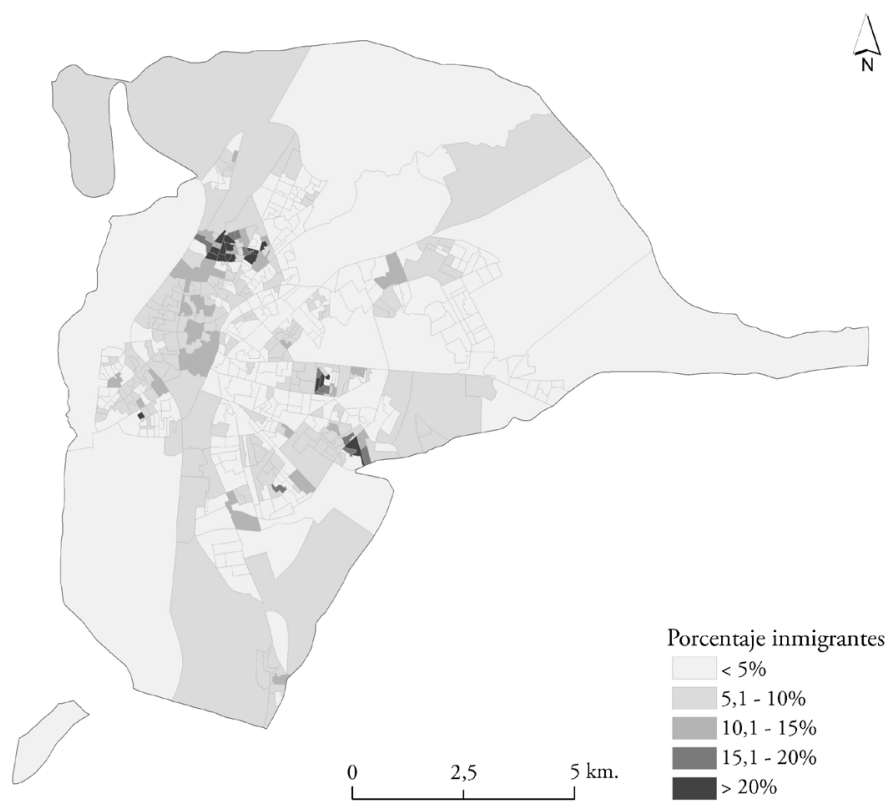

FUENTE SISTEMA DE INFORMACIÓN MULTITERRITORIAL DE ANDALUCÍA (SIMA), 2O I I. ELABORACIÓN PROPIA

FIGURA 2 Sevilla. Distritos y barrios que albergan secciones censales con más del $20 \%$ de población inmigrante, 2011

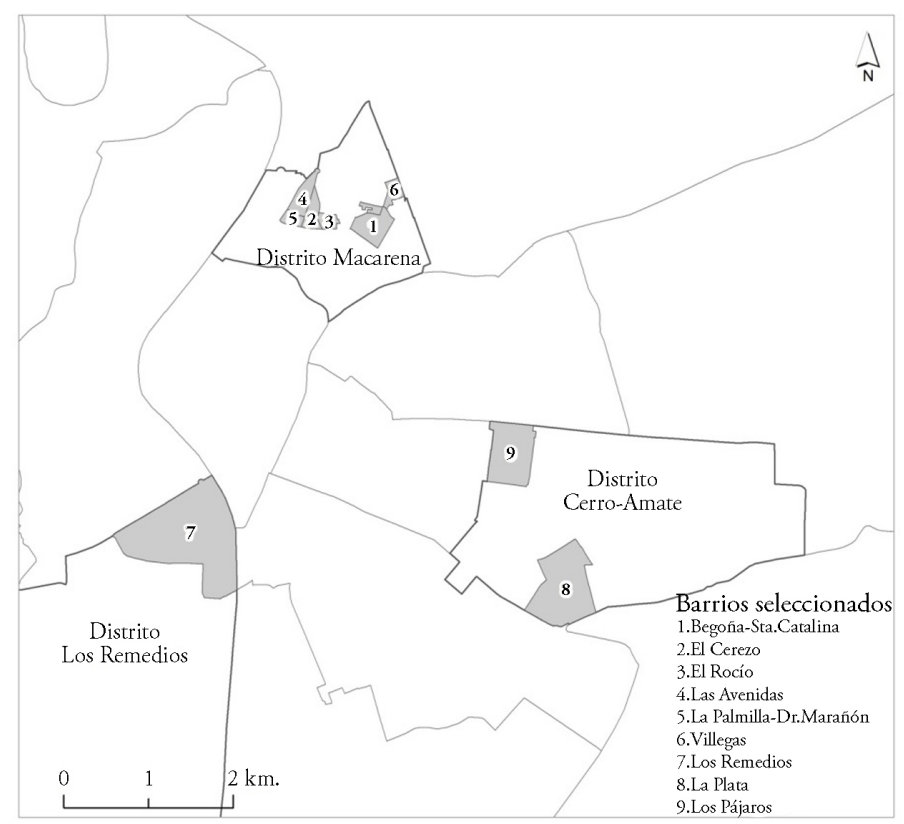

FUENTE

ELABORACIÓN PROPIA 
En concreto, se trata de los barrios El Rocío, El Cerezo, La Palmilla-Dr. Marañón, Begoña-Santa Catalina, Las Avenidas y Villegas, en el distrito dos de la ciudad (Macarena). En el distrito cuatro (Cerro-Amate), los barrios de Los Pájaros y La Plata; y por último, la sección censal tercera dentro del distrito once (Los Remedios). A partir de esta zonificación se analizó cómo construían los ciudadanos, a diferentes niveles de la escala metropolitana, su visión sobre aquellos barrios que albergan mayor presencia de inmigración extranjera y cómo determinaba dicho imaginario los procesos de segregación y estigmatización residencial.

\section{Metodología: desarrollando la territorialización del discurso}

Para comprender la estructura metodológica desarrollada, se debe tener en cuenta el discurso y las CES derivadas como constructo social colectivo que determina en gran medida las actitudes que los ciudadanos desarrollan hacia determinados ámbitos de la ciudad. Sobre esa base se orientó el análisis hacia la visión y los imaginarios desarrollados en el ámbito metropolitano de Sevilla respecto a aquellas secciones censales y barrios con mayor densidad de población inmigrante extranjera. Es en estas zonas donde primero aparecen las actitudes xenófobas, las cuales están íntimamente relacionadas con el filtrado residencial, que desemboca en procesos de segregación residencial de dicha población (Iglesias Pascual, 2015). Teniendo en cuenta la importancia de la dimensión intersubjetiva del objeto de análisis propuesto, se desarrolló lo que se ha denominado metodológicamente como la 'territorialización del discurso social'. Para ello, el instrumento central de recogida de información fue el de los grupos de discusión (a partir de ahora, GD). El desarrollo de GD se muestra como la estrategia más adecuada para la identificación de aquellas cogniciones sociales colectivas con las que socialmente se construye e interpreta la realidad social intersubjetiva (Alonso, 1996; Conde, 2009). Junto a los GD, la observación participante ha sido el otro mecanismo utilizado para la recogida de información. Ambos instrumentos metodológicos se pusieron en marcha en el mismo periodo de tiempo, en concreto entre septiembre, octubre y la primera quincena de noviembre de 2013. El motivo de acotar todo el trabajo de campo en un periodo tan específico fue evitar que cualquier aspecto social o mediático pudiese distorsionar de manera significativa las opiniones de los participantes.

\section{Caracterización y diseño de los grupos de discusión}

A la hora de diseñar los diferentes GD, se atendió especialmente al lugar de residencia de los participantes. Se buscaron componentes distribuidos en diferentes localizaciones del espacio metropolitano, que residiesen en zonas exteriores a los barrios seleccionados en la investigación. De este modo se plantea un análisis del discurso social para toda el área metropolitana que permita recoger las variaciones discursivas que en ella se dan según el lugar de residencia, para así valorar qué aspectos discursivos varían a lo largo del área metropolitana y cuáles son comunes a todo territorio. Para ello, a la hora de la realización de los GD se establecieron tres grandes zonas significativas del espacio metropolitano: la ciudad central o consolidada, y la primera y segunda corona metropolitana (figura 3). En cada una de estas zonas se 
desarrollaron diferentes GD para captar la intersubjetividad social de cada espacio y a la vez contrastar las diferencias entre los tres ámbitos señalados, con el objeto de alcanzar una visión global del discurso sobre la tipología de barrios seleccionada.

Junto con lo anterior, se consideró relevante realizar un análisis diferenciado del discurso según el estatus socioeconómico de la población en las diferentes escalas seleccionadas. Con tal fin, se dividieron los grupos en niveles socioeconómicos medio-bajo y medio-alto (Tezanos, 2004, pp. 348 y ss). Mediante esta división se buscaba conseguir una muestra de las actitudes de los diferentes grandes grupos socioeconómicos de la población de acogida respecto a los procesos de aceptación o rechazo de la población inmigrante. Los grupos definidos permiten una visión general respecto de cómo se desarrolla la subjetividad espacial en el ámbito metropolitano, así como de las posibles variaciones que se puedan producir dentro de él, por el lugar de residencia y las características socioeconómicas de los involucrados (tabla 1).

TABLA I Grupos de discusión realizados en el Área Metropolitana de Sevilla

\begin{tabular}{|c|l|l|}
\hline CóDIGO & \multicolumn{1}{|c|}{ ÁмBITo } & \multicolumn{1}{|c|}{ FECHA } \\
\hline GD 1 & Zona Centro Histórico. Estatus medio-alto & $15 / 10 / 2013$ \\
\hline GD 2 & Zona Macarena. Estatus medio-alto & $02 / 09 / 2013$ \\
\hline GD 3 & Zona Los Remedios. Estatus medio-alto & $22 / 10 / 2013$ (tarde) \\
\hline GD 4 & Zona Gran Plaza. Estatus medio-bajo & $27 / 09 / 2013$ \\
\hline GD 5 & 1a Corona (Dos Hermanas). Estatus medio-bajo & $08 / 09 / 2013$ \\
\hline GD 6 & 1 a Corona (Dos Hermanas). Estatus medio-alto & $10 / 09 / 2013$ \\
\hline GD 7 & 2a Corona (Utrera). Estatus medio-bajo & $28 / 09 / 2013$ \\
\hline GD 8 & 2a Corona (Utrera). Estatus medio-alto & $11 / 09 / 2013$ \\
\hline
\end{tabular}

FUENTE ELABORACIÓN PROPIA

A la importante fuente de información que suponen los GD, se añaden los diferentes registros tomados por el investigador respecto a las actitudes de los participantes antes, durante y especialmente tras la realización de las dinámicas grupales. El objetivo de esta última fase era analizar cómo trataban algunos de los temas abordados durante la dinámica grupal, de una manera más coloquial. Este doble discurso aporta una importante fuente de información a la hora de valorar el papel de lo implícito y lo oculto en los diferentes discursos individuales y colectivos (Martín Criado, 2014; Ruiz, 2014). La información así recogida fue especialmente esclarecedora en los grupos correspondientes al estatus medio-alto, donde se desarrollaba una mayor intención de matizar o encubrir algunos aspectos de sus planteamientos, buscando un discurso políticamente correcto (Cea D’Ancona, 2009a, p. 23). En el caso de los grupos correspondiente al estatus medio-bajo, en pocas ocasiones se observó grandes diferencias entre el discurso público en el GD y su posicionamiento posterior. 
FIgURA 3 | Distribución de los grupos de discusión en el Área Metropolitana de Sevilla

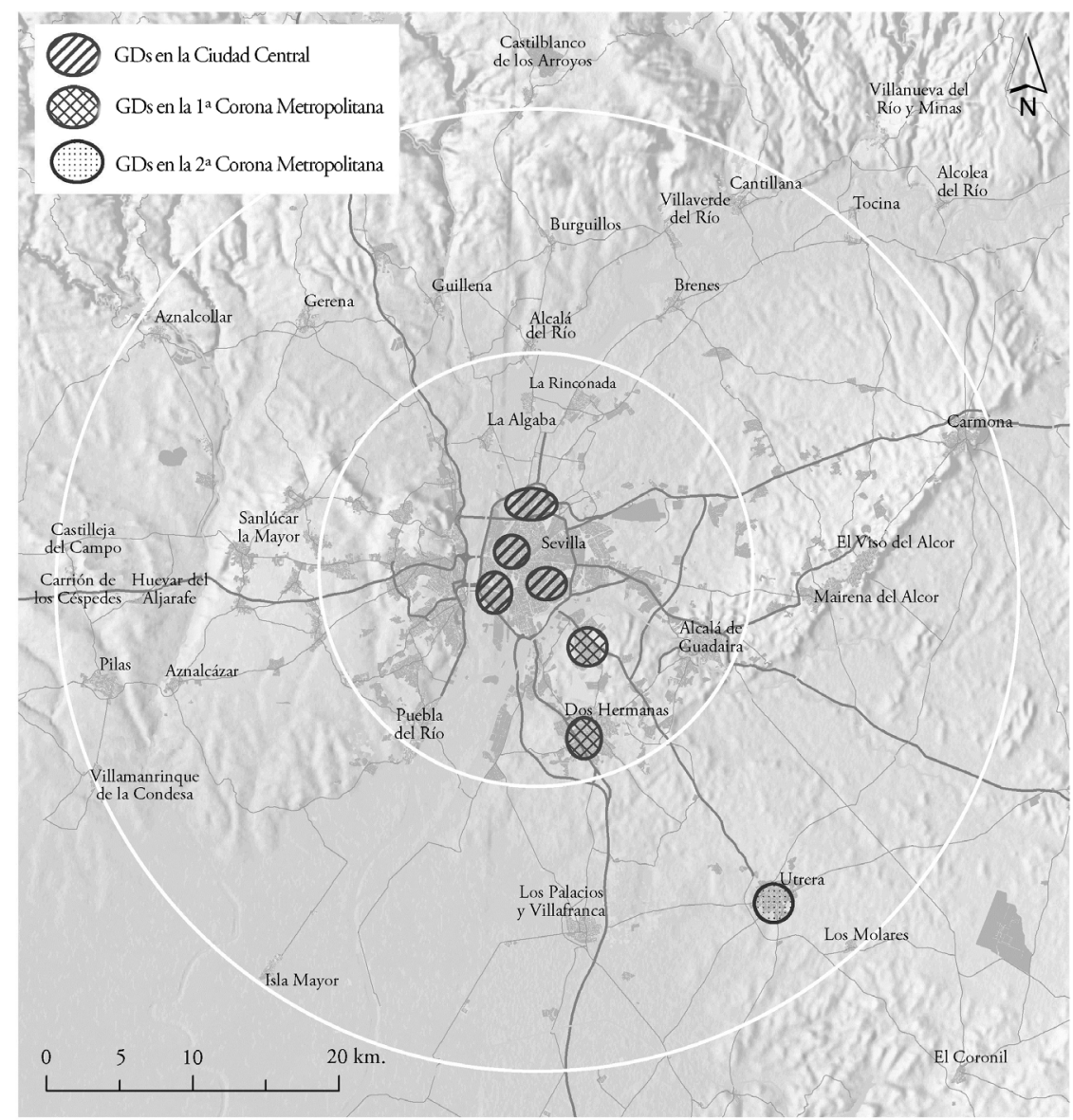

FUENTE ELABORACIÓN PROPIA A PARTIR DE ATLAS SEVILLA METROPOLITANA: TERRITORIO Y ACTIVIDADES PRODUCTIVAS (SEVILLA: SEVILla GLOBAL, 2003)

\section{Generadores del discurso}

Atendiendo a las principales líneas discursivas extraídas de los diferentes GD, fundamentalmente se pueden considerar tres de los principales generadores detectados en el origen del discurso sobre la inmigración y los barrios en los que se concentra. De manera generalizada, los tres elementos básicos referidos por los participantes como generadores de sus opiniones fueron los medios de comunicación, la visión interna de residentes o exresidentes en dichos barrios y, por último, los desplazamientos ocasionales (Iglesias Pascual, 2014). El mayor énfasis en uno u otro elemento variaba según la localización del GD y de los participantes en las diferentes escalas del espacio metropolitano. 
Junto con lo anterior, cabe destacar cómo los discursos construidos sobre los barrios seleccionados se pudieron encuadrar mayoritariamente dentro de las CES recreadas sobre espacios desconocidos o ignotos. Es decir, normalmente se genera la opinión sobre estos barrios sin prácticamente tener un conocimiento real sobre los mismos. Por tanto, su origen se encontraba en informaciones transmitidas de forma indirecta por los medios de comunicación o diferentes formas de interrelación social, pero sin conocerse de forma fehaciente el ámbito territorial sobre los que se han construido. Este aspecto reafirma la importancia de lo que se ha determinado como 'espacio inducido'.

La falta de conocimiento real sobre los barrios seleccionados hace que predominen las construcciones sociales basadas en ideas preconcebidas y estereotipadas, las cuales caracterizan en gran medida la dimensión inducida del espacio metropolitano. Tal como indica Cea D’Ancona (2009a), el predominio de informaciones indirectas, derivadas en gran medida de los medios de comunicación, acentúa las visiones estereotipadas sobre la población inmigrante. Al ser los barrios seleccionados para el estudio ámbitos con altas densidades de población inmigrante, la estigmatización de los inmigrantes se transmite al barrio donde residen, generalizándose unas CES que ponen el acento en una asociación de ideas que lleva desde el inmigrante a la inseguridad, al estigma y, de ahí, a la marginalización del barrio.

M3: Yo personalmente nunca he pasado por allí... lo asocio como algo peligroso por la noche. De día, un poquito extraño con gente de fuera, con una forma de vestir...

[GD 1. Zona Centro Histórico. Estatus medio-alto]

MI: Yo hablo de oídas.

v3: De todos modos, todos pasamos por allí en coche.

MI: Yo creo que los mismos sevillanos se encargan de dar esa sensación previa...

v2: Yo no he pasado por allí.

VI: Es de oídas.

M2: Casi siempre es de oídas primero, los nombres dicen mucho.

[GD 8. 2a Corona (Utrera). Estatus medio-alto]

\section{Los medios de comunicación}

En primer lugar, cabe destacar cómo, a la hora de referirse a este generador del discurso -los diferentes medios de comunicación-, los participantes lo hacen de una manera general, sin discriminar entre ellos. En el tratamiento sobre este aspecto, durante el desarrollo de los GD normalmente no especificaban qué medio presentaba mayor importancia en su papel como forjador de opiniones. El carácter difuso de las referencias discursivas respecto a los medios - en una sociedad que los consume, como indica Hartmut Rosa (2011) en su sociología de la aceleración, con una creciente rapidez e inmediatez, sin profundizar en su análisis (Ramonet, 1998) - muestra el carácter de visión estereotipada y basada en lugares comunes que 
suelen tener dichos discursos. Aunque se podría pensar que el principal forjador de opinión y estereotipos sociales es la televisión, a la hora de analizar las noticias de Sevilla y su entorno los diferentes GD mostraron cómo eran la radio y la prensa, debido a su carácter más local y cercano, los medios que desempeñaban un papel más activo en dicho proceso.

Sin duda la capacidad de influencia de los medios sobre la formación de opinión se entiende mejor teniendo en cuenta los principios de las denominadas teorías del agenda-setting (McCombs \& Shaw, 1972), priming (Fiske \& Taylor, 1984) y framing (D’Adamo, García Beaudoux \& Freidenberg, 2007). Dichas teorías analizan cómo los medios marcan claramente sobre qué pensar, cómo interpretar la información y el modo de interpretar los acontecimientos. Todos estos elementos inciden en gran manera en el constructo socioespacial en el que finalmente deviene el imaginario social que se desarrolla sobre el espacio metropolitano. Es decir, un espacio inducido que sirve como referencia a la hora de determinar las decisiones espaciales individuales y colectivas dentro de la ciudad.

VI: Es que hay sitios que no se puede pasar de Sevilla. Yo lo que conozco es por la prensa.

M2: Sí, yo también por los medios de comunicación...

[GD 6. $1^{\text {a }}$ Corona (Dos Hermanas). Estatus medio-alto]

MI: Yo no lo conozco de na', vamos de lo que he oído en la radio y cosas así.

M2: Eso... en las noticias y esas cosas.

M3: La tele...

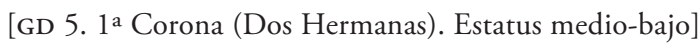

\section{La visión interna}

En esta categoría se aglutina la imagen transmitida por los mismos residentes o exresidentes de los barrios en los que se centra la investigación. La importancia dada a esta variable en los diferentes discursos hizo que se considerase necesario analizar cómo se construye dicha visión y cuáles son sus elementos más característicos. En este caso, el principal punto de interés fue determinar la autoimagen que estos barrios con presencia importante de inmigrantes desarrollan y transmiten al resto de la ciudad.

MI: Empecé a escuchar a través de una amiga que su hermana vive allí, los problemas que tienen... luego, bueno, periódicos, radio, etcétera.

M2: Yo a mí... como no es mi barrio, es una zona que no conozco, que no pateo, que no me afecta.

M3: Yo todas las referencias que tengo son de oídas; de vivencias, ninguna.

VI: Pues yo, las referencias que tengo es de una amiga que vive allí y que habla pestes de la convivencia. Dice que no se puede vivir allí, que traen unas costumbres de sus países y las practican aquí y esas costumbres hacen que sea imposible la convivencia.

[GD 3. Zona Los Remedios. Estatus medio-alto] 
Del análisis de los diferentes verbatims resultó especialmente reseñable el carácter normalmente negativo de las opiniones de los residentes o exresidentes que los miembros de los GD argumentaban principalmente como fuente de información. Con esto no se pretende indicar que la totalidad de las referencias que un individuo reciba de estos barrios sea negativa, pero sí se puede afirmar al menos que preferentemente seleccionan aquellas informaciones que reafirman los miedos, dudas e incertidumbres de los ciudadanos, más que aquellas que ponen en duda el discurso social y mediático dominante. El miedo al diferente -es decir, al extranjero-, al estar basado en gran medida en información generada a través experiencias indirectas, recibe un deseado refrendo de experiencias vitales reales conocidas a través de las opiniones de aquellos individuos que han vivido o viven en la zona. Dentro de esta categoría se pueden agrupar también los discursos de las personas cuya actividad laboral se localiza en estos barrios, a los que también se les da gran credibilidad.

\section{Desplazamientos ocasionales}

Este tercer elemento generador del discurso se muestra como una de las fuentes más fiables de información por parte de los participantes en las dinámicas grupales. En la mayoría de las ocasiones, estos desplazamientos son el momento de mayor cercanía real a las zonas sobre las que se construyen las CES, y de ahí la gran trascendencia que los participantes les otorgan. Al tratarse de una experiencia directa y vivida en primera persona, se les atribuye una gran veracidad. Sin embargo, se debe tener en cuenta que generalmente dichos desplazamientos se caracterizan por su carácter ocasional, rápido y poco profundo. La mayoría de los desplazamientos por barrios de alta densidad de inmigrantes se reducían a trayectos realizados en algún tipo de transporte público o preferentemente privado, caracterizados tanto por su rapidez como por la falta de interacción social. En estos casos, los pasos se limitaban a la duración del desplazamiento y la consecuente imagen que de este se derivaba.

V2: Tú, porque no te montas en el autobús; pero si te montas en el 1 o en el 2, y ahí te ves todos inmigrantes...

V3: ... y conduciendo por allí y observando, porque caminar por allí yo no camino... Que veo muchos negros por allí...

V2: Pasando por ahí...

MI: Yo, por comentarios de la gente, por lo que dicen por ahí...

[GD 4. Zona Gran Plaza. Estatus medio-bajo]

V2: En el autobús se ve mucho, se observa... te montas y lo ves llenos de inmigrantes.

M2: Para mí por mi trabajo, pregunto y siempre me dicen "cuidado hay mucho inmigrantes, familias desestructuradas..."

[GD 4. Zona Gran Plaza. Estatus medio-bajo] 
VI: ... yo pasé también una vez por allí con la moto y la verdad que aquello te daba la impresión de que era Harlem por la actitud desafiante en las puertas, los bares...

MI: Es que ellos son muy territoriales, traen unas costumbres muy territoriales. Nosotros hemos pasado por allí con la bici y en el carril bici te miran como diciendo "vosotros qué hacéis aquí metiendo las narices en mi barrio", y son desafiantes y se te quedan mirando...

[GD 3. Zona Los Remedios. Estatus medio-alto]

En un nivel de mayor interacción se pueden clasificar los desplazamientos relacionados con actividades laborales. En estos casos hay una mayor duración, pero al estar basados en trayectos concretos a localizaciones específicas, la interacción social por parte de los protagonistas con actores del barrio resulta generalmente escasa. Dentro de esta categoría también se pueden agrupar las visitas a familiares, centros sanitarios, actividades de ocio ocasionales, etcétera.

v3: Yo hace un año pasé por allí con el coche con mi mujer, que era de allí, y nos sorprendió la cantidad de...

MI: Yo las Tres Mil, porque cuando era estudiante porque apedreaban el tren al pasar.

V2: Yo cuando he ido a jugar al baloncesto....

[GD 6. $1^{\text {a }}$ Corona (Dos Hermanas). Estatus medio-alto]

En todos los casos se produce una estancia limitada en estos ámbitos, sin que se genere ni conocimiento ni interacción con los agentes sociales de los barrios. De hecho, en numerosas ocasiones se le da mayor importancia a las actitudes y comportamiento de la población extranjera, a la cual simplemente ven haciendo uso del espacio público o en comercios de otras nacionalidades y que en numerosas ocasiones califican como "desafiante". La sola presencia en las calles de población de otras nacionalidades, razas o culturas se considera como una agresión a la cohesión y convivencia del barrio. El uso del espacio público y de la calle, que hace visible al inmigrante extranjero, es asociado en el imaginario social con una invasión y, por tanto, se lo siente como una amenaza. En este sentido, diversas investigaciones cualitativas efectuadas en barrios españoles indican cómo en los estereotipos sobre las diferentes zonas residenciales, los varones jóvenes de origen extranjero se convierten en el signo más visible y potente de intimidación y delincuencia para los nativos (Echezarra, 2014; González Enríquez \& Álvarez-Miranda, 2005). Ello muestra el importante impacto que tienen en las actitudes hacia la inmigración el miedo a la diferencia y las consecuentes barreras simbólicas que se construyen como defensa ante lo desconocido.

En el caso de Sevilla, se debe tener en cuenta que el incremento de la población extranjera ha sido un hecho muy reciente, producido en un corto tiempo. ${ }^{4}$ Este

$4 \overline{\text { A pesar del bajo porcentaje de población extranjera en } 2011 \text { en Sevilla (5,4\%), su crecimiento }}$ 
carácter precipitado y acelerado de la llegada de nuevos miembros a la sociedad acrecienta los sentimientos de miedo e incertidumbre. Tales aspectos, de carácter subjetivo, se encontrarían en la base $e^{5}$ de los elevados valores de segregación residencial que presenta en Sevilla la población inmigrante, en comparación con otras ciudades con mayor presencia de dicha población.

Llegados a este punto, resulta importante señalar cómo, dentro de los GD realizados en la ciudad consolidada, solo el GD 3 hizo referencia a los medios de comunicación como elemento necesario de tener en cuenta a la hora de construir las opiniones sobre los barrios seleccionados. Este aspecto, tal como se comentó antes, contradice la hipótesis inicial de los estudios que plantean la importancia de los medios de comunicación como formadores del discurso sobre la inmigración. De hecho, a través del análisis del discurso se puede considerar que en entornos muy cercanos a las zonas de estudio, elementos como los pasos ocasionales y las informaciones obtenidas mediante otras personas presentan una mayor influencia social intersubjetiva que los medios de comunicación. Esto se acentúa en una ciudad como Sevilla, caracterizada por un fuerte nivel de cohesión interna e importancia de la tradición (Cachia, 2014), donde las referencias directas mediante la experiencia personal o los círculos de amistad tienen una decisiva influencia sobre las intersubjetividades sociales. Muestra de ello es la mayor importancia de los medios de comunicación en el espacio metropolitano de Sevilla a medida que se produce un alejamiento de la ciudad central, donde variables como la visión interna y los desplazamientos ocasionales son las que tienen mayor peso en los discursos.

Por tanto, se puede considerar que el mayor tamaño del espacio metropolitano produciría un aumento de la importancia de los medios de comunicación en detrimento de la visión interna o los desplazamientos ocasionales. Estos dos aspectos tendrían un carácter más esporádico y puntual en el espacio metropolitano, al encontrarse básicamente relacionados con las interacciones personales, las cuales se suponen más escasas al aumentar el tamaño del territorio analizado. Del mismo modo, ante el mayor tamaño del ámbito metropolitano se incrementa la dificultad de conocer la integridad del territorio, aumentando en importancia lo que se ha denominado como espacio inducido.

\section{La transversalidad territorial: el contraste entre los ámbitos metropolitanos}

Una vez analizados los principales elementos generadores del discurso, se abordan en los siguientes apartados aquellos aspectos claves para entender cómo se construyen las perspectivas discursivas existentes en las distintas escalas metropolitanas. De manera general, los tres generadores discursivos ya citados son las principales poleas de transmisión a la hora de construir las visiones sociales sobre la inmigración en el espacio metropolitano. Sin embargo, estos generadores no constituyen en sí

desde el ańo 2000 fue del 662\%.

5 Junto a otros aspectos como el precio de la vivienda, el estado del parque residencial o el reducido mercado de alquiler. 
mismos el discurso social respecto a la inmigración, el cual se plasma en una serie de categorías temáticas que se pueden considerar como transversales a todo el territorio.

Ahora bien, para entender la diferente intensidad y transversalidad con las que dichas temáticas aparecen en los discursos, se hace necesario analizar en primer lugar aquellos factores que influyen en su construcción: conocimiento de la ciudad, generadores del discurso y origen de los inmigrantes.

\section{El conocimiento de la ciudad}

Resulta en cierta medida obvio cómo el alejamiento de la ciudad central produce un mayor desconocimiento de sus diferentes barrios. Así, los GD realizados en esta parte de la ciudad muestran un alto conocimiento general del territorio y principales barrios históricos de Sevilla. A medida que se pasa de la primera a la segunda corona disminuye dicho conocimiento, independientemente del grupo socioeconómico al que se pertenezca.

A pesar de lo anterior, ciertos barrios y zonas de la ciudad quedan establecidos en el imaginario social colectivo como buenos barrios. Es el caso en Sevilla de zonas como Los Remedios, Nervión o el Centro Histórico. A su vez, otros barrios son percibidos tradicionalmente como ámbitos marginales, asociados principalmente con la delincuencia: tal sería el caso de Tres Mil Viviendas, Torreblanca, Los Pajaritos o Su Eminencia. Por último, cabe señalar otros ámbitos de la ciudad considerados como barrios invadidos por la inmigración, constructo social especialmente reservado para la zona de La Macarena.

Estos estereotipos territoriales sobre la ciudad central devienen en las grandes narraciones colectivas que se construyen sobre todo el espacio urbano y desempeñan un importante papel a la hora de determinar la movilidad y el filtrado residencial, especialmente en aquellos barrios con alta densidad de población inmigrante extranjera (Iglesias Pascual, 2016, p. 321). Es decir, son las CEs las que conforman el mapa mental individual y colectivo de la ciudad, especialmente a medida que aumenta la escala territorial. A mayor distancia respecto al espacio central de la ciudad, mayor dimensión alcanza el espacio inducido, convirtiéndose progresivamente en la principal dimensión espacial de referencia individual y colectiva.

\section{Variación en los generadores discursivos}

Como se ha comentado, el análisis del discurso social muestra cómo el aumento de la distancia territorial respecto a los barrios seleccionados incide en el mayor peso de los medios de comunicación respecto a los desplazamientos ocasionales y la visión de los residentes o exresidentes como generadores del discurso. Tal como se indica en la teoría del agenda setting propuesta por McCombs y Shaw (1972), la mayor parte del conocimiento que las personas poseen sobre los fenómenos sociales que acaecen a su alrededor no proviene de la experiencia personal directa, sino de los medios de comunicación (McCombs, 2004). De este modo, al aumentar la extensión del espacio urbano, los medios de comunicación se convierten en elemento fundamental a la hora de establecer las cuestiones relevantes que condicionan la aproximación de los actores a la realidad social de su entorno. Es decir, la ciudadanía metropolitana en 
gran medida conoce de su entorno lo que los medios le muestran, incrementándose dicha dependencia con la mayor extensión del espacio metropolitano.

\section{Nacionalidad, filias y fobias}

El origen de los inmigrantes y la actitud hacia ellos es otro de los aspectos que varía según el ámbito del espacio metropolitano en que se sitúe el GD analizado. Todos los grupos con que se trabajó mostraron actitudes diferenciadas ante las distintas nacionalidades. De este modo, la población extranjera residente en el distrito Centro Histórico, de origen mayoritariamente norteamericano y de la UE de los 15, no es denominada como inmigrante, sino como extranjera o "guiri". Esto refleja la incidencia social del denominado racismo de clases (Myrdal, 1944), ya que no es tanto la etnicidad como la inferior posición económica lo que explica los prejuicios hacia las diferentes nacionalidades. Tal racismo de clase prevalecería sobre el étnico y hace que en la sociedad española, tal como indica Díez Nicolás (2005), "el racismo y la xenofobia son, en la mayoría de los casos, expresión de un clasismo muy arraigado" (p. 189).

V2: Son internacionales.

VI: Eso pasa en la Alameda.

V2: Pero esos son italianos, hay franceses...

VI: Eso son ahora barrios "snobistas"... Hombre, no tienen la misma valoración, el poder adquisitivo es distinto.

V2: La gente que viene con gastos pagados o becarios... o vienen a estudiar, son gente joven que vienen a aprender idiomas y se van...

VI: Sí, no son gente que se quede aquí como los otros. Los otros quieren quedarse aquí o emigrar a Europa...

[GD 1. Zona Centro Histórico. Estatus medio-alto]

M I Los rumanos son los más problemáticos (en general).

V3: Y los chino', tío.

MI. Pa’ mi los rumanos y los chino', porque nos están invadiendo; es que hay un barrio prácticamente...

M2: Invadido por ellos.

[GD 7. 2a Corona (Utrera). Estatus medio-bajo]

\section{Discursos, temáticas y actitudes transversales a todo el territorio}

Una vez analizados los generadores del discurso sobre la inmigración y los elementos que intervienen en la construcción de las principales categorías temáticas que sobre la misma desarrolla la ciudadanía, en esta fase de la investigación se aborda el estudio de aquellas temáticas discursivas comunes a todo el territorio metropolitano. Estas temáticas, al difundirse transversalmente por la ciudad, se erigen en el elemento 
clave en la construcción de las distintas CEs, y en general del discurso intersubjetivo sobre la inmigración en la ciudad.

El análisis comparado de los discursos recogidos en las diferentes escalas metropolitanas muestra la fuerte influencia que las temáticas establecidas desde la ciudad central proyectan sobre el resto del espacio metropolitano. De este modo, se puede considerar que los discursos y actitudes desarrollados en las zonas más cercanas a los barrios seleccionados se ven apropiados en sus líneas más generales por el resto del espacio metropolitano, a pesar de que el conocimiento real sobre dichos ámbitos disminuye claramente en las sucesivas coronas metropolitanas. Esta apropiación del discurso y su carácter estereotipado inciden en la formación de las CES que se establecen en la ciudad respecto a los barrios con alta presencia de inmigrantes, influyendo en gran medida en la manera de ver y percibir esta tipología de barrios desde el conjunto de la ciudad. El discurso sobre la inmigración se genera localmente en puntos concretos de la ciudad, perpetuándose por el resto del territorio metropolitano como imaginarios sociales muy difíciles de revertir, que estigmatizan territorialmente aquellos barrios donde se concentra la inmigración.

Considerando lo anterior, se puede hablar de una proyección de las CEs de la ciudad central hacia la periferia, aunque con ligeras matizaciones, diferenciándose distintos niveles en el proceso. En primer lugar, destacan aquellas categorías temáticas y actitudes existentes en todos los ámbitos y todos los grupos de discusión realizados en la ciudad, por lo que se las ha denominado como transversalidad permanente. En segundo lugar se encuentran las categorías temáticas que, aunque detectadas en todos los ámbitos metropolitanos, no resultan generalizables a todos los GD. En este caso se observa cómo los elementos de estatus y lugar de residencia en el espacio metropolitano determinan el discurso. Dichas temáticas han sido adscritas bajo la denominación de transversalidad parcial. Por último, se ha establecido un nivel de transversalidad modulada para aquellas temáticas que pierden intensidad discursiva al aumentar la distancia respecto a la ciudad central, aunque no dejan de estar presentes en todos los ámbitos y GD.

\section{Transversalidad permanente}

Bajo esta categoría se han clasificado aquellas temáticas y actitudes que presentan una mayor trascendencia en los discursos de todos los GD, independientemente de su lugar en el espacio metropolitano. Se trata de aspectos comentados a lo largo del desarrollo de todas las dinámicas grupales de manera clara y generalizada, convertidos en una red de categorizaciones temáticas extendida por toda la ciudad.

- Los espacios de marginalidad: los barrios Los Pajaritos y Su Eminencia son concebidos de manera colectiva en todos los GD como espacios de marginalidad y delincuencia. Solo el GD 4 los identificó como espacios de fuerte presencia de población inmigrante, lo que se explica gracias a su cercanía geográfica con dichos barrios. La categorización como barrios marginales tiene su base en sus orígenes históricos (Torres, 2013) y se perpetúa constantemente en la ciudad a partir de referencias indirectas. 
- Locutorio y comercio, sinónimos de inmigración: esta asociación es común para todos grupos y ámbitos. La mera presencia de un locutorio - un centro que ofrece el servicio de llamadas telefónicas- supuso en todos los casos la aparición de referencias a la inmigración, aunque por lo general no se reconocía la zona donde estaban situados.

\section{Transversalidad parcial}

Dentro de esta categoría se agrupan aquellos aspectos generalizables a todo el espacio metropolitano, que presentan variaciones determinadas por el estatus social o el lugar del espacio de residencia dentro del área metropolitana.

- Macarena, zona invadida: el Distrito Macarena es percibido socialmente como una zona invadida por inmigrantes, personalizándose dicho imaginario especialmente en el barrio El Cerezo. Resulta destacable cómo esta categoría discursiva aparece únicamente en los GD realizados en la ciudad central y en los grupos de estatus medio-bajo de las coronas metropolitanas, resultando inexistente en los grupos de estatus medio-alto del ámbito metropolitano. En el caso de la ciudad consolidada, esto se debería a una mayor cercanía territorial a La Macarena, mientras que en los grupos de estatus medio-bajo se debe a una percepción de la inmigración como una competencia social por los recursos. En ambos casos se relaciona al inmigrante con la amenaza. Sin embargo, en los grupos de estatus medio-alto de ambas coronas metropolitanas, tanto la lejanía territorial como su situación económica hace que no perciban la inmigración como amenaza, desarrollando una visión de La Macarena como ámbito de clase media, muy determinado por su función sanitaria. ${ }^{6}$

- La importancia de la nacionalidad: como ya se ha indicado, la nacionalidad del inmigrante determina en gran manera la percepción que de él se tiene. Dichas visiones se relacionan tanto con el estatus socioeconómico como con la situación concreta del entorno donde se reside. A su vez, la nacionalidad predominante en cada ámbito territorial determina en gran manera las opiniones que sobre ella se desarrollan.

\section{Transversalidad modulada}

Las temáticas aquí encuadradas se caracterizan por variar en intensidad a medida que el análisis se acerca a la periferia del espacio metropolitano.

- Sensación de invasión: este aspecto resulta especialmente recurrente en los GD de la ciudad central, pero pierde fuerza a medida que se adentra en las coronas metropolitanas. Se mantiene principalmente en los grupos de estatus mediobajo y prácticamente va desapareciendo en el estatus superior, hasta convertirse en ligeras referencias al mismo. Esto se encuentra en consonancia con lo indicado por Cea D’Ancona (2008, 2009b), Cea D’Ancona y Valles (2010, 2011) respecto de cómo las actitudes racistas y xenófobas se incrementan en las zonas con mayor proporción de personas de distinta raza o etnia, ya sea en respuesta 
a una presencia real de ellas o como una respuesta a su presencia que ha sido transmitida y adquirida a través de relatos.

- La formación del discurso: a la hora de abordar la construcción de subjetividades e imaginarios relacionados con los barrios de mayor presencia de inmigrantes, ha quedado patente cómo varía la importancia de los tres generadores discursivos según la situación que se ocupe en el espacio metropolitano. De este modo, a mayor distancia respecto a los barrios analizados, mayor importancia adquieren los medios de comunicación en detrimento de las opiniones basadas en la movilidad ocasional y en relatos de residentes o exresidentes.

\section{Recapitulando: la reproducción del discurso sobre la inmigración y su potencialidad a la hora de diseńar políticas de integración}

El análisis desarrollado respecto del discurso social sobre la inmigración en Sevilla pone de manifiesto la importancia de su transversalidad territorial en el ámbito metropolitano, destacando especialmente la influencia ejercida por aquel que se genera desde la ciudad consolidada. Es decir, los discursos del área central se reflejan y reproducen de manera generalizada en todo el espacio metropolitano, a pesar de que el conocimiento sobre los barrios analizados disminuye claramente en las sucesivas coronas. De este modo, dicho discurso incide en la construcción de determinadas concepciones espaciales subjetivas (CES) desde las zonas más cercanas a los barrios con alta presencia de extranjeros que son apropiadas a grandes rasgos por el resto de la ciudad. Por tanto, el discurso y las CES recreadas localmente en puntos concretos de la ciudad se perpetúan y difunden por toda el área metropolitana como imaginarios sociales basados en el estereotipo y, por tanto, muy difíciles de revertir; es decir, se puede hablar de una proyección de las Ces de la ciudad consolidada hacia la periferia. En este sentido, en un nivel local y metropolitano como en el que se encuadra la investigación, los medios de comunicación, más que elementos creadores del discurso sobre el inmigrante, debieran ser clasificados como meros amplificadores del discurso más estereotipado y peyorativo generado hacia el inmigrante a nivel barrial.

Ante esta importancia de lo local en la formación del discurso sobre la inmigración, se pone de manifiesto la necesidad de dar prioridad a la escala microsocial a la hora de desarrollar las diferentes políticas de integración de las población inmigrante. En su diseño deben tenerse en cuenta tanto la importancia del discurso generado en el barrio que acoge al inmigrante y sus zonas aledańas, como su carácter estereotipado. Por tanto, desde un punto de vista territorial y a la luz del análisis realizado sobre los generadores y transversalidad del discurso sobre la inmigración y su comportamiento espacial en el área metropolitana, se pueden proponer dos grandes directrices a la hora de diseñar y aplicar medidas destinadas a la integración de la población inmigrante.

En primer lugar, partiendo de la creciente importancia que tiene la dimensión inducida del espacio urbano en las áreas metropolitanas, se plantea la necesidad de desarrollar una pedagogía de la ciudad. La dificultad de conocer completamente 
el territorio metropolitano suele generar reacciones caracterizadas por el miedo y la incertidumbre, convirtiéndose ambos en elementos fundamentales en la creación del discurso y el imaginario social. Miedo a lo desconocido, a lo diferente, a lo marginal. Un miedo producto del desconocimiento, elemento que del mismo modo se encuentra en la base de la aparición de actitudes xenófobas. Consecuentemente, si en el origen del discurso sobre los barrios con alta presencia de extranjeros se encuentran en gran medida el miedo, la percepción de inseguridad y la xenofobia, solo se puede actuar sobre estas creencias y opiniones mediante la educación y la información. Una educación territorial en este caso, que permita al ciudadano conocer las múltiples realidades de los barrios de su ciudad, sin caer en el carácter parcial y superficial que suelen utilizar los medios de comunicación. Por pedagogía territorial se puede entender una amplia gama de recursos que vayan desde programas de intercambio cultural en los barrios, organización de actividades deportivas de carácter itinerante por la ciudad, actuación en la escuela y centros de educación secundaria, actividades socializadoras de carácter multicultural, construcción de infraestructuras de ocio, etcétera. En resumen, todo aquello que ayude a hacer visible al ciudadano las diferentes unidades socioterritoriales de su ciudad.

En segundo lugar, como ha quedado demostrado a lo largo de la investigación, el discurso sobre la inmigración se construye a partir de informaciones secundarias e indirectas que suelen tener su origen en las narraciones que emanan del barrio o los pasos ocasionales que por ellos se producen. Por tanto, solo actuando sobre dichos mensajes se podrá revertir el carácter estereotipado del discurso. Es decir, si el discurso sobre la inmigración que se desarrolla en la ciudad es el fruto de la territorialización y cosificación de las opiniones que se originan en los barrios, debe actuarse mediante una acción informativa, directa y participativa que permita intervenir sobre el carácter estereotipado que lo caracteriza. En este sentido, proyectos de creación de una red ciudadana y agentes "antirrumores", como el desarrollado por el ayuntamiento de Barcelona desde $2010,{ }^{7}$ marcan un modelo de acción posible de llevar a cabo para facilitar la integración de la población inmigrante y prevenir la aparición de actitudes de tinte xenófobo o racista. Solo con una acción local, próxima al ciudadano, se puede combatir los estereotipos que se encuentran en la base del discurso y las percepciones construidas en relación con los inmigrantes.

\section{Referencias bibliográficas}

Alonso, L. E. (1996). El grupo de discusión en su práctica: memoria social, intertextualidad y acción comunicativa. Revista Internacional de Sociología, (13), 5-36.

Brown, L. \& Chung, S.Y . (2006). Spatial segregation, segregation indices and the geographical perspective. Population, Space and Place, 12(2), 125-143. doi: 10.1002/psp.403

7 El "proyecto antirrumores" lo aplica desde 2014 la Junta de Andalucía, así como las ciudades de Sabadell, Getxo, Fuenlabrada y la isla de Tenerife. Véase http://www.antirumores.com/ 
Cachia, R. (2014). Connected lives in contemporary mobile societies. Tesis doctoral en Psicología Social, dirigida por Isidro Maya Jariego. Universidad de Sevilla, Sevilla. https:// dialnet.unirioja.es/servlet/tesis? codigo $=54353$

Cea D’Ancona, M. Á. (2007). Inmigración, racismo y xenofobia en la España del nuevo contexto europeo. Madrid: Observatorio Espańol del Racismo y la Xenofobia (Oberaxe), Ministerio de Trabajo y Asuntos Sociales.

Cea D’Ancona, M. Á. (2009a). La compleja detección del racismo y la xenofobia a través de encuesta: un paso adelante en su medición. Revista Española de Investigaciones Sociológicas, (125), 13-46. http://www.reis.cis.es/REIS/PDF/ REIS_125_011231144723167.pdf

Cea D’Ancona, M. Á. (2009b). Filias y fobias ante la imagen poliédrica cambiante de la inmigración: claves en la comprensión del racismo y la xenofobia. Revista del Ministerio de Trabajo e Inmigración. Migraciones Internacionales, (80), 39-60. http://www. caritasvitoria.org/datos/documentos/filiasyfobias.pdf

Cea D’Ancona, M. Á. \& Valles Martínez, M. S. (2010). Living together: European citizenship against racism and xenophobia: Decalogue and final comparative and comprehensive report. Madrid: Observatorio Español del Racismo y la Xenofobia (Oberaxe), Ministerio de Trabajo e Inmigración.

Cea D’Ancona, M. Á. \& Valles Martínez, M. S. (2011). Evolución del racismo y la xenofobia en España: Informe 2011. Madrid: Observatorio Español del Racismo y la Xenofobia (Oberaxe), Ministerio de Trabajo e Inmigración.

Conde, F. (2009). Análisis sociológico del sistema de discursos. Madrid: Centro de Investigaciones Sociológicas.

D’ Adamo, O., García Beaudoux, V. \& Freidenberg, F. (2007). Medios de comunicación y opinión pública. Madrid: McGraw-Hill.

Díez Nicolás, J. (2005). Las dos caras de la inmigración. Madrid: Instituto de Mayores Servicios Sociales (Imserso), Ministerio de Trabajo y Asuntos Sociales. http://pendientedemigracion.ucm.es/info/gemi/descargas/libros/9DIEZ_NICOLASLas_Dos_Caras_ Inmigracion2005.pdf

Echazarra, A. (2014). La delincuencia en los barrios. Percepción y reacciones. Colección Estudios Sociales, 37. Barcelona: Obra Social La Caixa.

Farley, R., Steeh, C., Krysan, M., Jackso, T. \& Reeves, K. (1994). Stereotypes and segregation: Neighborhoods in the Detroit area. American Journal of Sociology, 100(3), 750-780. doi: $10.1086 / 230580$

Fiske, S. T. \& Taylor, S. E. (1984). Social cognition. Reading, MA: Addison-Wesley.

González Enríquez, C. \& Álvarez-Miranda, B. (2006). Inmigrantes en el barrio. Un estudio cualitativo de opinión pública. Documentos del Observatorio Permanente de la Inmigración, 6. Madrid: Ministerio de Trabajo y Asuntos Sociales. http://extranjeros. empleo.gob.es/es/ObservatorioPermanenteInmigracion/Publicaciones/fichas/ archivos/Inmigrantes_barrio.pdf 
Herranz de Rafael, G. (Investigador principal). Con Fernández, J. S., Rodríguez, P., Checa, J. C., Ainz, A., Fernández, I. \& Gonzalez, M. J. (2012). Xenofobia en Andalucía. Análisis en municipios y ciudades con alta densidad de inmigrantes. Sevilla: Fundación Pública Andaluza, Centro de Estudios Andaluces, Consejería de la Presidencia e Igualdad, Junta de Andalucía. https://www.centrodeestudiosandaluces.es/datos/factoriaideas/ IFO01_13.pdf

Igartua, J. J., Muñiz, C., Otero, J. A. \& De la Fuente, M. (2007). (2007). El tratamiento informativo de la inmigración en los medios de comunicación españoles. Un análisis de contenido desde la Teoría del Framing. Estudios sobre el Mensaje Periodístico, (13), 91-110. http://bit.ly/2hBC2fN

Igartua, J. J., Otero, J. A., Muñiz, C. \& Gómez, J. (2006). Efectos cognitivos y afectivos de los encuadres noticiosos de la inmigración. Ponencia presesentada en las $V$ Jornadas de Comunicación "Medios de Comunicación, Inmigración y Sociedad. Retos y propuestas para el siglo XX”, 7, 8 y 9 de marzo de 2006, Universidad de Salamanca, Salamanca. https://es.scribd.com/document/40306488/ Efectos-cognitivos-y-afectivos-de-los-encuadres-noticiosos-de-la-inmigracion

Iglesias Pascual, R. (2014). Generadores del discurso sobre inmigración extranjera y su difusión en el ámbito metropolitano. Ponencia presentada en el I Simposium Internacional EDISO. Estudios sobre Discurso y Sociedad.

Iglesias Pascual, R. (2015a). Segregación residencial, concepciones espaciales subjetivas y xenofobia en Sevilla. Determinando el umbral para la intervención social. Cuadernos Geográficos, 54(1), 230-256. http://revistaseug.ugr.es/index.php/cuadgeo/article/view/2525

Iglesias Pascual, R. (2015b). Segregación residencial y xenofobia. Utilizando la disrupción social para la gestión de la diversidad. En F. J. García Castaño, A. Megías Megías \& J. Ortega Torres (Eds.), Actas del VIII Congreso sobre Migraciones Internacionales en España (pp. S07/136-S07/149), Granada, Instituto de Migraciones, 16-18 de septiembre de 2015.

Iglesias Pascual, R. (2016). Espacio inducido y territorialización del discurso. Determinado el impacto socioterritorial del imaginario social sobre la inmigración en el Área Metropolitana de Sevilla. Documents d'Anàlisi Geogràfica, 62(2), 299-325. http://doi. org $/ 10.5565 / \mathrm{rev} / \mathrm{dag} .276$

Johnston, R. J., Poulsen, M. F. \& Forrest, J. (2002). Are there ethnic enclaves / ghettos in English cities? Urban Studies, 39(4), 591-618. doi: 10.1080/00420980220119480

Martín Criado, E. (2014). Mentiras, inconsistencias y ambivalencias. Teoría de la acción y análisis de discurso. Revista Internacional de Sociología, 72(1), 115-138. http://doi. org/10.3989/ris.2012.07.24

McCombs, M. E. (2004). Setting the agenda: The mass media and public opinion. Cambridg, UK: Polity Press.

McCombs, M. E. \& Shaw, D. L. (1972). The agenda-setting function of the mass media. Public Opinion Quarterly, 36(2), 176-185. https://www.unc.edu/ fbaum/teaching/ PLSC541_Fall06/McCombs\%20and\%20Shaw\%20POQ\%201972.pdf 
Myrdal, G. (1944). An American dilemma. The Negro problem and modern democracy. New York: Harper \& Brothers.

Palacios García, A. J. \& Vidal Domínguez, M. J. (2014). La distribución intraurbana de los inmigrantes en las ciudades espańolas: un análisis de casos con SIG y técnicas cuantitativas. Cuadernos Geográficos, 53(1), 98-121. http://revistaseug.ugr.es/index. $\mathrm{php/cuadgeo/article/view/1335}$

Ramonet, I. (1998). La tiranía de la comunicación. Madrid: Debate.

Rosa, H. (2011). Aceleración social: consecuencias éticas y políticas de una sociedad de alta velocidad desincronizada. Persona y Sociedad, 25(1), 9-49. http://personaysociedad. cl/ojs/index.php/pys/article/view/161

Ruiz, J. (2014). El discurso implícito: aportaciones para un análisis sociológico. Revista Española de Investigaciones Sociológicas, (146), 171-190. doi:10.5477/cis/reis.146.171

Tezanos, J. F. (2004). La sociedad dividida: estructuras de clases y desigualdades en las sociedades tecnológicas. Madrid: Biblioteca Nueva.

Torres, F. J. (2013). Segregación urbana y exclusión social en Sevilla. El paradigma Polígono Sur. Sevilla: Universidad de Sevilla.

Van Dijk, T. A. (2003). Racismo y discurso de las élites. Barcelona: Gedisa.

Vono, D. \& Bayona, J. (2011). El asentamiento residencial de los latinoamericanos en las principales ciudades españolas (2001-2009). Notas de Población, 37(91), 129-159. Santiago, Chile: Comisión Económica para América Latina y el Caribe (Cepal). http://repositorio.cepal.org/bitstream/handle/11362/37691/1/S1000525_es.pdf

Wacquant, L. (2007). Los condenados de la ciudad: gueto, periferias, Estado. Buenos Aires: Siglo XXI. 